K. Kojo

Nagoya Math. J.

Vol. 180 (2005), 135-149

\title{
TWO-DIMENSIONAL SYMMETRIC STABLE DISTRIBUTIONS AND THEIR PROJECTIONS
}

\author{
KATSUYA KOJO
}

\begin{abstract}
We study the problem whether a given 2-dimensional symmetric stable distribution with index $\alpha(0<\alpha \leq 1)$ is determined by its 1-dimensional projections in some specified directions. We give some conditions for the affirmative answer and for the negative answer.
\end{abstract}

\section{$\S 1$. Introduction and preliminaries}

An $\mathbf{R}^{d}$-valued random variable $X=\left(X_{1}, X_{2}, \ldots, X_{d}\right)$ is said to be stable if for any $A, B>0$, there exist $C>0$ and $D \in \mathbf{R}$ such that

$$
A X^{(1)}+B X^{(2)} \stackrel{d}{=} C X+D \quad(\stackrel{d}{=} \text { means equality in distribution })
$$

where $X^{(1)}$ and $X^{(2)}$ are independent copies of $X$. If $X$ is stable and non-constant, then there exists a constant $\alpha(0<\alpha \leq 2)$ such that $C=$ $\left(A^{\alpha}+B^{\alpha}\right)^{1 / \alpha}$ and therefore $X$ is called $\alpha$-stable $(\alpha$ is called the index of stability of $X) . X$ is called strictly stable if (1.1) holds with $D=0$ for any $A, B>0 . X$ is called symmetric stable if $X$ is stable and satisfies $-X \stackrel{d}{=} X$. An $\mathbf{R}$-valued random variable $X$ is symmetric $\alpha$-stable $(0<\alpha \leq 2)$ if and only if $E \exp (i z X)=\exp \left(-c|z|^{\alpha}\right), z \in \mathbf{R}$, for some $c \geq 0$. Especially, when $\alpha=2, X$ is Gaussian with mean 0 . An $\mathbf{R}^{d}$-valued random variable $X$ is $d$-dimensional symmetric $\alpha$-stable $(0<\alpha<2)$ if and only if

$$
\begin{aligned}
E \exp \left(i \sum_{j=1}^{d} z_{j} X_{j}\right)=\exp \left(-\int_{\xi=\left(\xi_{1}, \xi_{2}, \ldots, \xi_{d}\right) \in S^{d-1}}\left|\sum_{j=1}^{d} z_{j} \xi_{j}\right|^{\alpha} \Gamma(d \xi)\right) & \\
z & =\left(z_{1}, z_{2}, \ldots, z_{d}\right) \in \mathbf{R}^{d},
\end{aligned}
$$

for some symmetric finite measure $\Gamma$ on the $(d-1)$-dimensional unit sphere $S^{d-1}$. This $\Gamma$ is uniquely determined by the distribution of $X$ and is called the spectral measure of $X$.

Received July 23, 2004.

2000 Mathematics Subject Classification: 60E07. 
The problem whether an $\mathbf{R}^{d}$-valued random variable $X=\left(X_{1}, X_{2}, \ldots\right.$, $\left.X_{d}\right)$ is stable if all 1-dimensional projections $\sum_{j=1}^{d} z_{j} X_{j}, z_{j} \in \mathbf{R}$, are stable is studied variously. An $\mathbf{R}^{d}$-valued random variable is symmetric stable (respectively, strictly stable) if and only if all 1-dimensional projections are symmetric stable (respectively, strictly stable) (see Theorem 2.1.5 in G. Samorodnitsky and S. Taqqu [2]). If $1 \leq \alpha \leq 2$, an $\mathbf{R}^{d}$-valued random variable is $\alpha$-stable if and only if all 1 -dimensional projections are $\alpha$-stable. However, if $0<\alpha<1$, there exists a non-stable $\mathbf{R}^{2}$-valued random variable such that all 1-dimensional projections are $\alpha$-stable (D. J. Marcus [1]).

Two $\mathbf{R}^{d}$-valued random variables $X=\left(X_{1}, X_{2}, \ldots, X_{d}\right)$ and $\tilde{X}=$ $\left(\tilde{X}_{1}, \tilde{X}_{2}, \ldots, \tilde{X}_{d}\right)$ are identically distributed if $\sum_{j=1}^{d} z_{j} X_{j} \stackrel{d}{=} \sum_{j=1}^{d} z_{j} \tilde{X}_{j}$ for all $z_{j} \in \mathbf{R}$. In the case where two 2-dimensional random variables $X=\left(X_{1}, X_{2}\right)$ and $\tilde{X}=\left(\tilde{X}_{1}, \tilde{X}_{2}\right)$ are Gaussian with mean 0 , they are identically distributed if $\left(\cos \theta_{k}\right) X_{1}+\left(\sin \theta_{k}\right) X_{2} \stackrel{d}{=}\left(\cos \theta_{k}\right) \tilde{X}_{1}+\left(\sin \theta_{k}\right) \tilde{X}_{2}$, $k=1,2,3$, for some $\theta_{1}, \theta_{2}$ and $\theta_{3}\left(0 \leq \theta_{1}<\theta_{2}<\theta_{3}<\pi\right)$. In this paper we study the problem whether a given 2-dimensional symmetric $\alpha$-stable distribution $(0<\alpha \leq 1)$ is determined by its 1 -dimensional projections in some specified directions. In Section 2, we see that for any 2-dimensional symmetric $\alpha$-stable random variable $X=\left(X_{1}, X_{2}\right)(0<\alpha \leq 1)$, there exists a 2-dimensional symmetric $\alpha$-stable random variable $\tilde{X}=\left(\tilde{X}_{1}, \tilde{X}_{2}\right)$ such that $z_{1} \tilde{X}_{1}+z_{2} \tilde{X}_{2} \stackrel{d}{=} z_{1} X_{1}+z_{2} X_{2}$ for uncountably many pairs $\left(z_{1}, z_{2}\right)$ with $z_{1}^{2}+z_{2}^{2}=1$ although $\tilde{X} \stackrel{d}{\neq} X$. In Section 3 , we see that for a certain 2 -dimensional symmetric $\alpha$-stable random variable $X=\left(X_{1}, X_{2}\right)(0<\alpha<$ 1 ), there does not exist a 2-dimensional symmetric $\alpha$-stable random variable $\tilde{X}=\left(\tilde{X}_{1}, \tilde{X}_{2}\right)$ such that $\tilde{X} \stackrel{d}{\neq} X$ and $z_{1} \tilde{X}_{1}+z_{2} \tilde{X}_{2} \stackrel{d}{=} z_{1} X_{1}+z_{2} X_{2}$ for some specified vectors $\left(z_{1}, z_{2}\right) \in \mathbf{R}^{2}$.

\section{$\S 2$. The existence of distribution with common projections in uncountably many directions}

Henceforth we identify the unit circle $S^{1}$ with $[0,2 \pi)$ and denote $\xi_{1}=$ $\cos \xi, \xi_{2}=\sin \xi$ for $\xi \in[0,2 \pi)$.

Theorem 2.1. Let $X=\left(X_{1}, X_{2}\right)$ be a 2-dimensional symmetric $\alpha$ stable random variable $(0<\alpha \leq 1)$. If the spectral measure $\Gamma$ of $X$ satisfies $\Gamma((0, \pi / 2))>0$, then there exists a 2-dimensional symmetric $\alpha$-stable ran- 
dom variable $\tilde{X}=\left(\tilde{X}_{1}, \tilde{X}_{2}\right)$ satisfying $\tilde{X} \stackrel{d}{\neq} X$ such that

$$
z_{1} \tilde{X}_{1}+z_{2} \tilde{X}_{2} \stackrel{d}{=} z_{1} X_{1}+z_{2} X_{2} \quad \text { if } z_{1} z_{2} \geq 0
$$

Proof. First we consider the case $\Gamma([\pi / 2, \pi])=0$. If there exists an $\mathbf{R}^{2}$-valued random variable $\tilde{X}=\left(\tilde{X}_{1}, \tilde{X}_{2}\right)$ such that

$$
\begin{aligned}
& E \exp \left(i\left(z_{1} \tilde{X}_{1}+z_{2} \tilde{X}_{2}\right)\right) \\
& = \begin{cases}\exp \left(-\int_{[0,2 \pi)}\left|z_{1} \xi_{1}+z_{2} \xi_{2}\right|^{\alpha} \Gamma(d \xi)\right) & \text { if } z_{1} z_{2} \geq 0, \\
\exp \left(-\int_{[0,2 \pi)}\left|z_{1} \xi_{1}-z_{2} \xi_{2}\right|^{\alpha} \Gamma(d \xi)\right) & \text { if } z_{1} z_{2}<0,\end{cases}
\end{aligned}
$$

then $\tilde{X}$ is symmetric $\alpha$-stable and satisfies the condition (C1). Further, we have $\tilde{X} \stackrel{d}{\neq} X$ since $\Gamma((0, \pi / 2))>0$ and $\left|\xi_{1}+\xi_{2}\right|^{\alpha}>\left|\xi_{1}-\xi_{2}\right|^{\alpha}$ for $\xi \in(0, \pi / 2)$. Therefore we have only to show the existence of $\tilde{X}$.

Let $\tilde{\varphi}\left(z_{1}, z_{2}\right)$ denote the right hand side of $(2.1)$. Let $\epsilon \in(0, \pi / 4)$ be a constant with $\Gamma((\epsilon, \pi / 2-\epsilon))>0$ and we have

$$
\begin{aligned}
c & =\int_{\mathbf{R}^{2}} \tilde{\varphi}\left(z_{1}, z_{2}\right) d z_{1} d z_{2} \\
& =2 \int_{z_{1}>0, z_{2}>0} \tilde{\varphi}\left(z_{1}, z_{2}\right) d z_{1} d z_{2}+2 \int_{z_{1}>0, z_{2}<0} \tilde{\varphi}\left(z_{1}, z_{2}\right) d z_{1} d z_{2} \\
& =4 \int_{z_{1}>0, z_{2}>0} \exp \left(-2 \int_{[0, \pi)}\left|z_{1} \xi_{1}+z_{2} \xi_{2}\right|^{\alpha} \Gamma(d \xi)\right) d z_{1} d z_{2} \\
& \leq 4 \int_{z_{1}>0, z_{2}>0} \exp \left(-2 \int_{(\epsilon, \pi / 2-\epsilon)}\left|z_{1} \xi_{1}+z_{2} \xi_{2}\right|^{\alpha} \Gamma(d \xi)\right) d z_{1} d z_{2} \\
& \leq 4 \int_{z_{1}>0, z_{2}>0} \exp \left(-c_{1}\left|z_{1}\right|^{\alpha}-c_{2}\left|z_{2}\right|^{\alpha}\right) d z_{1} d z_{2}<\infty
\end{aligned}
$$

for some $c_{1}, c_{2}>0$. Since $\tilde{\varphi}\left(z_{1}, z_{2}\right)$ is positive, $\tilde{\varphi}\left(z_{1}, z_{2}\right) / c$ is a density function of a distribution on $\mathbf{R}^{2}$. Let $f\left(x_{1}, x_{2}\right)$ be

$$
f\left(x_{1}, x_{2}\right)=\frac{1}{(2 \pi)^{2}} \int_{\mathbf{R}^{2}} \exp \left(-i\left(x_{1} z_{1}+x_{2} z_{2}\right)\right) \tilde{\varphi}\left(z_{1}, z_{2}\right) d z_{1} d z_{2} .
$$

Then we have

$$
\frac{1}{c} \int_{\mathbf{R}^{2}} \exp \left(i\left(x_{1} z_{1}+x_{2} z_{2}\right)\right) \tilde{\varphi}\left(z_{1}, z_{2}\right) d z_{1} d z_{2}=\frac{(2 \pi)^{2}}{c} f\left(x_{1}, x_{2}\right),
$$


using $\tilde{\varphi}\left(-z_{1},-z_{2}\right)=\tilde{\varphi}\left(z_{1}, z_{2}\right)$. Suppose that we have shown that $\int_{\mathbf{R}^{2}}\left|f\left(x_{1}, x_{2}\right)\right| d x_{1} d x_{2}<\infty$. Then we have

$$
\frac{1}{c} \tilde{\varphi}\left(z_{1}, z_{2}\right)=\frac{1}{(2 \pi)^{2}} \int_{\mathbf{R}^{2}} \exp \left(-i\left(x_{1} z_{1}+x_{2} z_{2}\right)\right) \frac{(2 \pi)^{2}}{c} f\left(x_{1}, x_{2}\right) d x_{1} d x_{2}
$$

by inverse Fourier transform and therefore

$$
\tilde{\varphi}\left(z_{1}, z_{2}\right)=\int_{\mathbf{R}^{2}} \exp \left(i\left(x_{1} z_{1}+x_{2} z_{2}\right)\right) f\left(x_{1}, x_{2}\right) d x_{1} d x_{2},
$$

using $f\left(-x_{1},-x_{2}\right)=f\left(x_{1}, x_{2}\right)$. If we further show that $f\left(x_{1}, x_{2}\right)$ is nonnegative, we find that $\tilde{\varphi}\left(z_{1}, z_{2}\right)$ is the characteristic function of a distribution with density $f\left(x_{1}, x_{2}\right)$. That is, $\tilde{X}$ exists.

Let us show that $f\left(x_{1}, x_{2}\right)>0$. The function $f\left(x_{1}, x_{2}\right)$ can be written as

$$
\begin{aligned}
f\left(x_{1}, x_{2}\right)=\frac{1}{\pi^{2}} & \int_{0}^{\infty} \cos \left(x_{2} z_{2}\right) \int_{0}^{\infty} \cos \left(x_{1} z_{1}\right) \\
& \times \exp \left(-2 \int_{(0, \pi / 2)}\left(z_{1} \xi_{1}+z_{2} \xi_{2}\right)^{\alpha} \Gamma(d \xi)\right) d z_{1} d z_{2} .
\end{aligned}
$$

We note that if a function $g:(0, \infty) \rightarrow \mathbf{R}$ is summable and $g^{\prime \prime}>0$ on $(0, \infty)$, then $\int_{0}^{\infty} g(x) \cos k x d x>0$ for any $k \in \mathbf{R}$. Let us define

$$
I_{x_{1}}\left(z_{2}\right)=\int_{0}^{\infty} \cos \left(x_{1} z_{1}\right) \exp \left(-2 \int_{(0, \pi / 2)}\left(z_{1} \xi_{1}+z_{2} \xi_{2}\right)^{\alpha} \Gamma(d \xi)\right) d z_{1} .
$$

Then

$$
\frac{d^{2}}{d z_{2}^{2}} I_{x_{1}}\left(z_{2}\right)=\int_{0}^{\infty} \cos \left(x_{1} z_{1}\right) \frac{\partial^{2}}{\partial z_{2}^{2}} \exp \left(-2 \int_{(0, \pi / 2)}\left(z_{1} \xi_{1}+z_{2} \xi_{2}\right)^{\alpha} \Gamma(d \xi)\right) d z_{1} .
$$

We find that

$$
\begin{aligned}
& \frac{\partial^{2}}{\partial z_{2}{ }^{2}} \exp \left(-2 \int_{(0, \pi / 2)}\left(z_{1} \xi_{1}+z_{2} \xi_{2}\right)^{\alpha} \Gamma(d \xi)\right) \\
&=\exp \left(-2 \int_{(0, \pi / 2)}\left(z_{1} \xi_{1}+z_{2} \xi_{2}\right)^{\alpha} \Gamma(d \xi)\right) \\
& \times\left\{\left(2 \alpha \int_{(0, \pi / 2)}\left(z_{1} \xi_{1}+z_{2} \xi_{2}\right)^{\alpha-1} \xi_{2} \Gamma(d \xi)\right)^{2}\right. \\
&\left.-2 \alpha(\alpha-1) \int_{(0, \pi / 2)}\left(z_{1} \xi_{1}+z_{2} \xi_{2}\right)^{\alpha-2} \xi_{2}^{2} \Gamma(d \xi)\right\}
\end{aligned}
$$


is summable on $(0, \infty)$ with respect to $z_{1}$ for any fixed $z_{2}>0$ because

$$
\begin{aligned}
\left|\frac{\partial^{2}}{\partial z_{2}{ }^{2}} \exp \left(-2 \int_{(0, \pi / 2)}\left(z_{1} \xi_{1}+z_{2} \xi_{2}\right)^{\alpha} \Gamma(d \xi)\right)\right| \\
\leq \exp \left(-2 \int_{(0, \pi / 2)}\left(z_{1} \xi_{1}+z_{2} \xi_{2}\right)^{\alpha} \Gamma(d \xi)\right) \\
\times\left\{\left(2 \alpha \int_{(0, \pi / 2)}\left(z_{2} \xi_{2}\right)^{\alpha-1} \xi_{2} \Gamma(d \xi)\right)^{2}\right. \\
\left.+2 \alpha(1-\alpha) \int_{(0, \pi / 2)}\left(z_{2} \xi_{2}\right)^{\alpha-2} \xi_{2}^{2} \Gamma(d \xi)\right\} .
\end{aligned}
$$

And we obtain that $\frac{\partial^{2}}{\partial z_{1}^{2}} \frac{\partial^{2}}{\partial z_{2}^{2}} \exp \left(-2 \int_{(0, \pi / 2)}\left(z_{1} \xi_{1}+z_{2} \xi_{2}\right)^{\alpha} \Gamma(d \xi)\right)>0$ by direct calculations, so that $\frac{d^{2}}{d z_{2}^{2}} I_{x_{1}}\left(z_{2}\right)$ is positive for any $x_{1}$. Moreover, $I_{x_{1}}\left(z_{2}\right)$ is summable for any $x_{1}$ and therefore we find $f\left(x_{1}, x_{2}\right)>0$.

We write $I=\int_{\mathbf{R}^{2}} f\left(x_{1}, x_{2}\right) d x_{1} d x_{2}$. Although we need only to show that $I<\infty$, let us show that $I=1$. We have

$$
\begin{aligned}
& I= \lim _{k_{1}, k_{2} \rightarrow \infty} \int_{-k_{2}}^{k_{2}} \int_{-k_{1}}^{k_{1}} f\left(x_{1}, x_{2}\right) d x_{1} d x_{2} \\
&=\frac{1}{\pi^{2}} \lim _{k_{1}, k_{2} \rightarrow \infty} \int_{0}^{\infty} \int_{0}^{\infty} \exp \left(-2 \int_{(0, \pi / 2)}\left(z_{1} \xi_{1}+z_{2} \xi_{2}\right)^{\alpha} \Gamma(d \xi)\right) d z_{1} d z_{2} \\
& \quad \times \int_{-k_{2}}^{k_{2}} \int_{-k_{1}}^{k_{1}} \cos \left(x_{1} z_{1}\right) \cos \left(x_{2} z_{2}\right) d x_{1} d x_{2} \\
&=\frac{4}{\pi^{2}} \lim _{k_{1}, k_{2} \rightarrow \infty} \int_{0}^{\infty} \int_{0}^{\infty} \frac{\sin \left(k_{1} z_{1}\right)}{z_{1}} \frac{\sin \left(k_{2} z_{2}\right)}{z_{2}} \\
& \quad \times \frac{\exp \left(-2 \int_{(0, \pi / 2)}\left(z_{1} \xi_{1}+z_{2} \xi_{2}\right)^{\alpha} \Gamma(d \xi)\right) d z_{1} d z_{2}}{\pi^{2}} \lim _{k_{1}, k_{2} \rightarrow \infty}^{\infty} \int_{0}^{\infty} \frac{\sin z_{1}}{z_{1}} \frac{\sin z_{2}}{z_{2}} \\
& \quad \times \exp \left(-2 \int_{(0, \pi / 2)}\left(\frac{z_{1}}{k_{1}} \xi_{1}+\frac{z_{2}}{k_{2}} \xi_{2}\right)^{\alpha} \Gamma(d \xi)\right) d z_{1} d z_{2} \\
&=\frac{4}{\pi^{2}} \lim _{k_{1}, k_{2} \rightarrow \infty} \sum_{m, n=0}^{\infty} \int_{2 n \pi}^{2(n+1) \pi} \int_{2 m \pi}^{2(m+1) \pi} \sin z_{1} \sin z_{2} \\
& \times \frac{1}{z_{1} z_{2}} \exp \left(-2 \int_{(0, \pi / 2)}\left(\frac{z_{1}}{k_{1}} \xi_{1}+\frac{z_{2}}{k_{2}} \xi_{2}\right)^{\alpha} \Gamma(d \xi)\right) d z_{1} d z_{2} .
\end{aligned}
$$


Here we define $q_{k_{1}, k_{2}}\left(z_{1}, z_{2}\right)=\frac{1}{z_{1} z_{2}} \exp \left(-2 \int_{(0, \pi / 2)}\left(\frac{z_{1}}{k_{1}} \xi_{1}+\frac{z_{2}}{k_{2}} \xi_{2}\right)^{\alpha} \Gamma(d \xi)\right)$ and $\tilde{Q}\left(k_{1}, k_{2}, z_{1}, z_{2}, w_{1}, w_{2}\right)=q_{k_{1}, k_{2}}\left(z_{1}, z_{2}\right)-q_{k_{1}, k_{2}}\left(w_{1}, z_{2}\right)-q_{k_{1}, k_{2}}\left(z_{1}, w_{2}\right)+$ $q_{k_{1}, k_{2}}\left(w_{1}, w_{2}\right)$. For $w_{1}>z_{1}$ and $w_{2}>z_{2}$, we find $\tilde{Q}\left(k_{1}, k_{2}, z_{1}, z_{2}, w_{1}, w_{2}\right)>0$ because

$$
\begin{aligned}
& \frac{\partial}{\partial w_{1}} \tilde{Q}\left(k_{1}, k_{2}, z_{1}, z_{2}, w_{1}, w_{2}\right) \\
&=\frac{1}{w_{1}^{2}}\left\{\frac{1}{z_{2}} \exp \left(-2 \int_{(0, \pi / 2)}\left(\frac{w_{1}}{k_{1}} \xi_{1}+\frac{z_{2}}{k_{2}} \xi_{2}\right)^{\alpha} \Gamma(d \xi)\right)\right. \\
&\left.\quad-\frac{1}{w_{2}} \exp \left(-2 \int_{(0, \pi / 2)}\left(\frac{w_{1}}{k_{1}} \xi_{1}+\frac{w_{2}}{k_{2}} \xi_{2}\right)^{\alpha} \Gamma(d \xi)\right)\right\} \\
&+\frac{2 \alpha}{w_{1}}\left\{\frac{1}{z_{2}} \exp \left(-2 \int_{(0, \pi / 2)}\left(\frac{w_{1}}{k_{1}} \xi_{1}+\frac{z_{2}}{k_{2}} \xi_{2}\right)^{\alpha} \Gamma(d \xi)\right)\right. \\
&\left.\left.\quad-\frac{w_{1}}{w_{2}} \xi_{1}+\frac{z_{2}}{k_{2}} \xi_{2}\right)^{\alpha-1} \frac{\xi_{1}}{k_{1}} \Gamma(d \xi)\right) \\
&\left.\quad-2 \int_{(0, \pi / 2)}\left(\frac{w_{1}}{k_{1}} \xi_{1}+\frac{w_{2}}{k_{2}} \xi_{2}\right)^{\alpha} \Gamma(d \xi)\right) \\
&>0
\end{aligned}
$$

and $\frac{\partial}{\partial w_{2}} \tilde{Q}\left(k_{1}, k_{2}, z_{1}, z_{2}, w_{1}, w_{2}\right)>0$. We define $Q_{k_{1}, k_{2}}\left(z_{1}, z_{2}\right)=q_{k_{1}, k_{2}}\left(z_{1}, z_{2}\right)$ $-q_{k_{1}, k_{2}}\left(z_{1}+\pi, z_{2}\right)-q_{k_{1}, k_{2}}\left(z_{1}, z_{2}+\pi\right)+q_{k_{1}, k_{2}}\left(z_{1}+\pi, z_{2}+\pi\right)$, then we have

$$
I=\frac{4}{\pi^{2}} \lim _{k_{1}, k_{2} \rightarrow \infty} \sum_{m, n=0}^{\infty} \int_{2 n \pi}^{(2 n+1) \pi} \int_{2 m \pi}^{(2 m+1) \pi} \sin z_{1} \sin z_{2} Q_{k_{1}, k_{2}}\left(z_{1}, z_{2}\right) d z_{1} d z_{2} .
$$

By $Q_{k_{1}, k_{2}}\left(z_{1}, z_{2}\right)=\tilde{Q}\left(k_{1}, k_{2}, z_{1}, z_{2}, z_{1}+\pi, z_{2}+\pi\right)>0$, we obtain $I_{m, n, k_{1}, k_{2}}=$ $\int_{2 n \pi}^{(2 n+1) \pi} \int_{2 m \pi}^{(2 m+1) \pi} \sin z_{1} \sin z_{2} Q_{k_{1}, k_{2}}\left(z_{1}, z_{2}\right) d z_{1} d z_{2}>0$. And we have

$$
\begin{aligned}
& \frac{\partial}{\partial k_{1}} \tilde{Q}\left(k_{1}, k_{2}, z_{1}, z_{2}, w_{1}, w_{2}\right) \\
&=\frac{2 \alpha}{k_{1}^{2}}\left\{\frac { 1 } { z _ { 2 } } \left(\exp \left(-2 \int_{(0, \pi / 2)}\left(\frac{z_{1}}{k_{1}} \xi_{1}+\frac{z_{2}}{k_{2}} \xi_{2}\right)^{\alpha} \Gamma(d \xi)\right)\right.\right. \\
& \times\left(\int_{(0, \pi / 2)}\left(\frac{z_{1}}{k_{1}} \xi_{1}+\frac{z_{2}}{k_{2}} \xi_{2}\right)^{\alpha-1} \xi_{1} \Gamma(d \xi)\right)
\end{aligned}
$$




$$
\begin{aligned}
&-\exp \left(-2 \int_{(0, \pi / 2)}\left(\frac{w_{1}}{k_{1}} \xi_{1}+\frac{z_{2}}{k_{2}} \xi_{2}\right)^{\alpha} \Gamma(d \xi)\right)\left.\left(\int_{(0, \pi / 2)}\left(\frac{w_{1}}{k_{1}} \xi_{1}+\frac{z_{2}}{k_{2}} \xi_{2}\right)^{\alpha-1} \xi_{1} \Gamma(d \xi)\right)\right) \\
&-\frac{1}{w_{2}}\left(\exp \left(-2 \int_{(0, \pi / 2)}\left(\frac{z_{1}}{k_{1}} \xi_{1}+\frac{w_{2}}{k_{2}} \xi_{2}\right)^{\alpha} \Gamma(d \xi)\right)\right. \\
& \quad \times\left(\int_{(0, \pi / 2)}\left(\frac{z_{1}}{k_{1}} \xi_{1}+\frac{w_{2}}{k_{2}} \xi_{2}\right)^{\alpha-1} \xi_{1} \Gamma(d \xi)\right) \\
&-\exp \left(-2 \int_{(0, \pi / 2)}\left(\frac{w_{1}}{k_{1}} \xi_{1}+\frac{w_{2}}{k_{2}} \xi_{2}\right)^{\alpha} \Gamma(d \xi)\right) \\
&\left.\left.\times\left(\int_{(0, \pi / 2)}\left(\frac{w_{1}}{k_{1}} \xi_{1}+\frac{w_{2}}{k_{2}} \xi_{2}\right)^{\alpha-1} \xi_{1} \Gamma(d \xi)\right)\right)\right\}
\end{aligned}
$$

We find that

$$
\begin{aligned}
& \exp \left(-2 \int_{(0, \pi / 2)}\left(\frac{z_{1}}{k_{1}} \xi_{1}+\frac{z_{2}}{k_{2}} \xi_{2}\right)^{\alpha} \Gamma(d \xi)\right) \\
& -\exp \left(-2 \int_{(0, \pi / 2)}\left(\frac{w_{1}}{k_{1}} \xi_{1}+\frac{z_{2}}{k_{2}} \xi_{2}\right)^{\alpha} \Gamma(d \xi)\right) \\
& >\exp \left(-2 \int_{(0, \pi / 2)}\left(\frac{z_{1}}{k_{1}} \xi_{1}+\frac{w_{2}}{k_{2}} \xi_{2}\right)^{\alpha} \Gamma(d \xi)\right) \\
& -\exp \left(-2 \int_{(0, \pi / 2)}\left(\frac{w_{1}}{k_{1}} \xi_{1}+\frac{w_{2}}{k_{2}} \xi_{2}\right)^{\alpha} \Gamma(d \xi)\right), \\
& \int_{(0, \pi / 2)}\left(\frac{z_{1}}{k_{1}} \xi_{1}+\frac{z_{2}}{k_{2}} \xi_{2}\right)^{\alpha-1} \xi_{1} \Gamma(d \xi) \\
& \quad-\int_{(0, \pi / 2)}\left(\frac{w_{1}}{k_{1}} \xi_{1}+\frac{z_{2}}{k_{2}} \xi_{2}\right)^{\alpha-1} \xi_{1} \Gamma(d \xi) \\
& \int_{(0, \pi / 2)}\left(\frac{z_{1}}{k_{1}} \xi_{1}+\frac{w_{2}}{k_{2}} \xi_{2}\right)^{\alpha-1} \xi_{1} \Gamma(d \xi) \\
& -\int_{(0, \pi / 2)}\left(\frac{w_{1}}{k_{1}} \xi_{1}+\frac{w_{2}}{k_{2}} \xi_{2}\right)^{\alpha-1} \xi_{1} \Gamma(d \xi)
\end{aligned}
$$

using that $x^{\alpha}(0<\alpha \leq 1)$ is concave and $x^{\alpha-1}(0<\alpha<1)$ and $e^{-x}$ are convex for $x>0$. We note that if $A_{1}, A_{2}, A_{3}, A_{4}, B_{1}, B_{2}, B_{3}$ and $B_{4}$ are positive numbers such that $A_{1}>A_{3}, B_{2}>B_{4}, A_{1}-A_{2}>A_{3}-A_{4}>0$ 
and $B_{1}-B_{2}>B_{3}-B_{4}>0$, then $A_{1} B_{1}-A_{2} B_{2}=A_{1}\left(B_{1}-B_{2}\right)+\left(A_{1}-\right.$ $\left.A_{2}\right) B_{2}>A_{3}\left(B_{3}-B_{4}\right)+\left(A_{3}-A_{4}\right) B_{4}=A_{3} B_{3}-A_{4} B_{4}$. Therefore we find $\frac{\partial}{\partial k_{1}} \tilde{Q}\left(k_{1}, k_{2}, z_{1}, z_{2}, w_{1}, w_{2}\right), \frac{\partial}{\partial k_{2}} \tilde{Q}\left(k_{1}, k_{2}, z_{1}, z_{2}, w_{1}, w_{2}\right)>0$, so that $I_{m, n, k_{1}, k_{2}}$ monotonously increases in $k_{1}, k_{2}$. Hence we obtain

$$
\begin{aligned}
I= & \frac{4}{\pi^{2}} \sum_{m, n=0}^{\infty} \lim _{k_{1}, k_{2} \rightarrow \infty} \int_{2 n \pi}^{(2 n+1) \pi} \int_{2 m \pi}^{(2 m+1) \pi} \sin z_{1} \sin z_{2} Q_{k_{1}, k_{2}}\left(z_{1}, z_{2}\right) d z_{1} d z_{2} \\
= & \frac{4}{\pi^{2}} \sum_{m, n=0}^{\infty} \int_{2 n \pi}^{2(n+1) \pi} \int_{2 m \pi}^{2(m+1) \pi} \frac{\sin z_{1}}{z_{1}} \frac{\sin z_{2}}{z_{2}} \\
& \times \lim _{k_{1}, k_{2} \rightarrow \infty} \exp \left(-2 \int_{(0, \pi / 2)}\left(\frac{z_{1}}{k_{1}} \xi_{1}+\frac{z_{2}}{k_{2}} \xi_{2}\right)^{\alpha} \Gamma(d \xi)\right) d z_{1} d z_{2} \\
& =\frac{4}{\pi^{2}} \int_{0}^{\infty} \frac{\sin z_{1}}{z_{1}} d z_{1} \int_{0}^{\infty} \frac{\sin z_{2}}{z_{2}} d z_{2} \\
= & 1 .
\end{aligned}
$$

Next we consider the case $\Gamma([\pi / 2, \pi])>0$. Let $\Gamma_{1}, \Gamma_{2}$ be

$$
\begin{aligned}
& \Gamma_{1}(B)=\Gamma(B \cap((0, \pi / 2) \cup(\pi, 3 \pi / 2))), \\
& \Gamma_{2}(B)=\Gamma(B \cap([\pi / 2, \pi] \cup[3 \pi / 2,2 \pi])) \text { for a Borel set } B \subset[0,2 \pi) .
\end{aligned}
$$

Then $\Gamma=\Gamma_{1}+\Gamma_{2}$. Let us define $\tilde{X}=\left(\tilde{X}_{1}, \tilde{X}_{2}\right)$ such that

$$
\begin{aligned}
& E \exp \left(i\left(z_{1} \tilde{X}_{1}+z_{2} \tilde{X}_{2}\right)\right) \\
& =\left\{\begin{array}{r}
\exp \left(-\int_{S^{1}}\left|z_{1} \xi_{1}+z_{2} \xi_{2}\right|{ }^{\alpha} \Gamma_{1}(d \xi)\right) \exp \left(-\int_{S^{1}}\left|z_{1} \xi_{1}+z_{2} \xi_{2}\right|{ }^{\alpha} \Gamma_{2}(d \xi)\right) \\
\text { if } z_{1} z_{2} \geq 0, \\
\exp \left(-\int_{S^{1}}\left|z_{1} \xi_{1}-z_{2} \xi_{2}\right|{ }^{\alpha} \Gamma_{1}(d \xi)\right) \exp \left(-\int_{S^{1}}\left|z_{1} \xi_{1}+z_{2} \xi_{2}\right|{ }^{\alpha} \Gamma_{2}(d \xi)\right) \\
\text { if } z_{1} z_{2}<0 .
\end{array}\right.
\end{aligned}
$$

We can easily see that there exists a 2-dimensional symmetric stable random variable whose characteristic function is $\exp \left(-\int_{S^{1}}\left|z_{1} \xi_{1}+z_{2} \xi_{2}\right|{ }^{\alpha} \Gamma_{1}(d \xi)\right)$ if $z_{1} z_{2} \geq 0$, and $\exp \left(-\int_{S^{1}}\left|z_{1} \xi_{1}-z_{2} \xi_{2}\right|^{\alpha} \Gamma_{1}(d \xi)\right)$ if $z_{1} z_{2}<0$. Its spectral measure is denoted by $\tilde{\Gamma}_{1}$ and we have $E \exp \left(i\left(z_{1} \tilde{X}_{1}+z_{2} \tilde{X}_{2}\right)\right)=\exp \left(-\int_{S^{1}} \mid z_{1} \xi_{1}+\right.$ $\left.\left.z_{2} \xi_{2}\right|^{\alpha}\left(\tilde{\Gamma}_{1}+\Gamma_{2}\right)(d \xi)\right)$. Obviously, $\tilde{\Gamma}_{1}+\Gamma_{2}$ satisfies the properties of spectral measures and we find that $\tilde{X}$ exists and satisfies the condition (C1). 
We note that, if $\alpha>1$, any symmetric $\alpha$-stable random variable has a differentiable characteristic function. Therefore, if $\alpha>1, \Gamma((0, \pi / 2))>0$ and $\Gamma([\pi / 2, \pi])=0$, then the right hand side of $(2.1), \tilde{\varphi}\left(z_{1}, z_{2}\right)$, cannot be the characteristic function of a symmetric $\alpha$-stable random variable, because $\tilde{\varphi}\left(z_{1}, z_{2}\right)$ is non-differentiable on $z_{1}=0$ and $z_{2}=0$.

Theorem 2.1 can be extended to the following proposition. Here, if $\left(z_{1}, z_{2}\right) \in \mathbf{R}^{2} \backslash\{(0,0)\}$ is written as $z_{1}=r \cos \theta, z_{2}=r \sin \theta(r>0)$, then $\theta$ is denoted by $\arg \left(z_{1}, z_{2}\right)$.

Proposition 2.2. Let $X=\left(X_{1}, X_{2}\right)$ be a 2-dimensional symmetric $\alpha$-stable random variable $(0<\alpha \leq 1)$ whose spectral measure $\Gamma$ satisfies $\Gamma\left(\left(\theta_{1}, \theta_{2}\right)\right)>0\left(0 \leq \theta_{1}<\theta_{2}<\pi\right)$. Then there exists a 2-dimensional symmetric $\alpha$-stable random variable $\tilde{X}=\left(\tilde{X}_{1}, \tilde{X}_{2}\right)$ satisfying $\tilde{X} \stackrel{d}{\neq} X$ such that

$$
z_{1} \tilde{X}_{1}+z_{2} \tilde{X}_{2} \stackrel{d}{=} z_{1} X_{1}+z_{2} X_{2} \quad \text { if } \arg \left(z_{1}, z_{2}\right) \in\left[\theta_{2}-\pi / 2, \theta_{1}+\pi / 2\right] .
$$

Proof. Let $Y=\left(Y_{1}, Y_{2}\right)$ be a 2-dimensional symmetric stable random variable such that

$$
\left\{\begin{array}{l}
Y_{1}=X_{1} \sin \theta_{2}-X_{2} \cos \theta_{2} \\
Y_{2}=-X_{1} \sin \theta_{1}+X_{2} \cos \theta_{1}
\end{array}\right.
$$

Then

$$
\begin{aligned}
& E \exp \left(i\left(z_{1} Y_{1}+z_{2} Y_{2}\right)\right) \\
& =E \exp \left(i\left(z_{1}\left(X_{1} \sin \theta_{2}-X_{2} \cos \theta_{2}\right)+z_{2}\left(-X_{1} \sin \theta_{1}+X_{2} \cos \theta_{1}\right)\right)\right) \\
& =E \exp \left(i\left(\left(z_{1} \sin \theta_{2}-z_{2} \sin \theta_{1}\right) X_{1}-\left(z_{1} \cos \theta_{2}-z_{2} \cos \theta_{1}\right) X_{2}\right)\right) \\
& =\exp \left(-\int_{[0,2 \pi)} \mid\left(z_{1} \sin \theta_{2}-z_{2} \sin \theta_{1}\right) \cos \xi\right. \\
& \left.\quad-\left.\left(z_{1} \cos \theta_{2}-z_{2} \cos \theta_{1}\right) \sin \xi\right|^{\alpha} \Gamma(d \xi)\right) \\
& =\exp \left(-\int_{[0,2 \pi)} \mid z_{1}\left(\sin \theta_{2} \cos \xi-\cos \theta_{2} \sin \xi\right)\right. \\
& \left.\quad+\left.z_{2}\left(\sin \xi \cos \theta_{1}-\cos \xi \sin \theta_{1}\right)\right|^{\alpha} \Gamma(d \xi)\right) \\
& =\exp \left(-\int_{[0,2 \pi)}\left|z_{1} \sin \left(\theta_{2}-\xi\right)+z_{2} \sin \left(\xi-\theta_{1}\right)\right|^{\alpha} \Gamma(d \xi)\right) .
\end{aligned}
$$


Here let us define the function $\sigma: \xi \in[0,2 \pi) \rightarrow \eta \in[0,2 \pi)$ such that

$$
\left\{\begin{array}{l}
\cos \eta=\sin \left(\theta_{2}-\xi\right) / \sqrt{\sin ^{2}\left(\theta_{2}-\xi\right)+\sin ^{2}\left(\xi-\theta_{1}\right)} \\
\sin \eta=\sin \left(\xi-\theta_{1}\right) / \sqrt{\sin ^{2}\left(\theta_{2}-\xi\right)+\sin ^{2}\left(\xi-\theta_{1}\right)} .
\end{array}\right.
$$

We note that the function $\sigma$ is one-to-one correspondence. Therefore we have

$$
E \exp \left(i\left(z_{1} Y_{1}+z_{2} Y_{2}\right)\right)=\exp \left(-\int_{[0,2 \pi)}\left|z_{1} \cos \eta+z_{2} \sin \eta\right|^{\alpha} \Gamma_{Y}(d \eta)\right)
$$

where $\Gamma_{Y}(d \eta)$ is the spectral measure of $Y$ and satisfies $\Gamma_{Y}(d \eta)=\left(\sin ^{2}\left(\theta_{2}-\right.\right.$ $\left.\left.\sigma^{-1}(\eta)\right)+\sin ^{2}\left(\sigma^{-1}(\eta)-\theta_{1}\right)\right)^{\alpha / 2} \Gamma\left(d \sigma^{-1}(\eta)\right)$. We see that $\eta=\sigma(\xi)$ changes from 0 to $\pi / 2$ as $\xi$ changes from $\theta_{1}$ to $\theta_{2}$ and therefore $\Gamma_{Y}((0, \pi / 2))>0$. By Theorem 2.1, there exists a 2-dimensional symmetric stable random variable $\tilde{Y}=\left(\tilde{Y}_{1}, \tilde{Y}_{2}\right)$ such that $z_{1} \tilde{Y}_{1}+z_{2} \tilde{Y}_{2} \stackrel{d}{=} z_{1} Y_{1}+z_{2} Y_{2}$ for $z_{1} z_{2} \geq 0$ although $\tilde{Y} \stackrel{d}{\neq} Y$.

Let us define a 2-dimensional symmetric stable random variable $\tilde{X}=$ $\left(\tilde{X}_{1}, \tilde{X}_{2}\right)$ such that

$$
\left\{\begin{array}{l}
\tilde{Y}_{1}=\tilde{X}_{1} \sin \theta_{2}-\tilde{X}_{2} \cos \theta_{2} \\
\tilde{Y}_{2}=-\tilde{X}_{1} \sin \theta_{1}+\tilde{X}_{2} \cos \theta_{1} .
\end{array}\right.
$$

Let $w=\left(w_{1}, w_{2}\right)$ be $w_{1}=z_{1} \sin \theta_{2}-z_{2} \sin \theta_{1}$ and $w_{2}=-z_{1} \cos \theta_{2}+z_{2} \cos \theta_{1}$. Then we can easily see that $w_{1} \tilde{X}_{1}+w_{2} \tilde{X}_{2} \stackrel{d}{=} w_{1} X_{1}+w_{2} X_{2}$ for $z_{1} z_{2} \geq 0$. When $z_{2}=0$ and $z_{1}>0$, we have $w_{1}=z_{1} \sin \theta_{2}$ and $w_{2}=-z_{1} \cos \theta_{2}$ so that $\arg w=\theta_{2}-\pi / 2$. When $z_{1}=0$ and $z_{2}>0$, we have $w_{1}=-z_{2} \sin \theta_{1}$ and $w_{2}=z_{2} \cos \theta_{1}$ so that $\arg w=\theta_{1}+\pi / 2$. Since the linear transformation from $\left(z_{1}, z_{2}\right)$ to $\left(w_{1}, w_{2}\right)$ has a positive determinant, we obtain that $\arg w$ changes from $\theta_{2}-\pi / 2$ to $\theta_{1}+\pi / 2$ for $z_{1}, z_{2} \geq 0$. Hence we find that $w_{1} \tilde{X}_{1}+w_{2} \tilde{X}_{2} \stackrel{d}{=} w_{1} X_{1}+w_{2} X_{2}$ for $\arg w \in\left[\theta_{2}-\pi / 2, \theta_{1}+\pi / 2\right]$. Obviously $\tilde{X} \stackrel{d}{\neq} X$.

Proposition 2.2 implies an interesting corollary as follows.

Corollary 2.3. For any non-zero 2-dimensional symmetric $\alpha$-stable random variable $X=\left(X_{1}, X_{2}\right)(0<\alpha \leq 1)$, there exists a 2-dimensional symmetric $\alpha$-stable random variable $\tilde{X}=\left(\tilde{X}_{1}, \tilde{X}_{2}\right)$ such that $z_{1} \tilde{X}_{1}+z_{2} \tilde{X}_{2} \stackrel{d}{=}$ $z_{1} X_{1}+z_{2} X_{2}$ for uncountably many pairs $\left(z_{1}, z_{2}\right)$ with $z_{1}^{2}+z_{2}^{2}=1$ although $\tilde{X} \stackrel{d}{\neq} X$ 


\section{$\S 3$. The distribution determined by its projections in some spec- ified directions}

As for Theorem 2.1, in the case $\Gamma((0, \pi / 2))=0$, we have an example with the opposite property.

Proposition 3.1. Let $X=\left(X_{1}, X_{2}\right)$ be a 2-dimensional symmetric $\alpha$-stable random variable $(0<\alpha<1)$ whose spectral measure $\Gamma$ satisfies that $\left.\Gamma\right|_{[0, \pi)}$ is concentrated on at most three points on $[\pi / 2, \pi)$. Suppose that a 2-dimensional symmetric $\alpha$-stable random variable $\tilde{X}=\left(\tilde{X}_{1}, \tilde{X}_{2}\right)$ satisfies the condition $(\mathrm{C} 1)$. Then $\tilde{X} \stackrel{d}{=} X$.

Proof. We handle the case where $\left.\Gamma\right|_{[0, \pi)}$ is supported on exactly three points in $[\pi / 2, \pi)$. (In the case where it is supported on one or two points, the proof is similar.) Namely we assume that $\Gamma\left(\left\{\theta_{j}\right\}\right)=p_{j}>0(j=1,2,3)$ and $\Gamma\left([0, \pi) \backslash\left\{\theta_{1}, \theta_{2}, \theta_{3}\right\}\right)=0$ for $\pi / 2 \leq \theta_{1}<\theta_{2}<\theta_{3}<\pi$. Calculating the characteristic function of 1-dimensional projection of $X$, we have

$$
\begin{aligned}
- & \frac{1}{2} \log E \exp \left(i\left((\cos \theta) X_{1}+(\sin \theta) X_{2}\right)\right) \\
& =\int_{[0, \pi)}|\cos \theta \cos \xi+\sin \theta \sin \xi|^{\alpha} \Gamma(d \xi)=\int_{[0, \pi)}|\cos (\theta-\xi)|^{\alpha} \Gamma(d \xi) .
\end{aligned}
$$

Suppose that $\tilde{X}$ is a 2-dimensional symmetric $\alpha$-stable random variable which satisfies the condition $(\mathrm{C} 1)$ and let $\tilde{\Gamma}$ be the spectral measure of $\tilde{X}$. If $\theta=\theta_{j}-\pi / 2(j=1,2,3)$, then we have $(\cos \theta) \tilde{X}_{1}+(\sin \theta) \tilde{X}_{2} \stackrel{d}{=}$ $(\cos \theta) X_{1}+(\sin \theta) X_{2}$. Therefore we have

$$
\begin{aligned}
\int_{[0, \pi)}\left|\sin \left(\theta_{j}-\xi\right)\right|^{\alpha} \tilde{\Gamma}(d \xi)=\left|\sin \left(\theta_{j}-\theta_{1}\right)\right|^{\alpha} p_{1} \\
\quad+\left|\sin \left(\theta_{j}-\theta_{2}\right)\right|^{\alpha} p_{2}+\left|\sin \left(\theta_{j}-\theta_{3}\right)\right|^{\alpha} p_{3}, \quad j=1,2,3
\end{aligned}
$$

If $\theta=\theta_{j}-\pi / 2+\epsilon(j=1,2,3)$ for sufficiently small $\epsilon>0$, then we also have $(\cos \theta) \tilde{X}_{1}+(\sin \theta) \tilde{X}_{2} \stackrel{d}{=}(\cos \theta) X_{1}+(\sin \theta) X_{2}$. Therefore we have

$$
\begin{aligned}
\int_{[0, \pi)} \mid & \left.\sin \left(\theta_{j}+\epsilon-\xi\right)\right|^{\alpha} \tilde{\Gamma}(d \xi)=\left|\sin \left(\theta_{j}-\theta_{1}+\epsilon\right)\right|^{\alpha} p_{1} \\
& +\left|\sin \left(\theta_{j}-\theta_{2}+\epsilon\right)\right|^{\alpha} p_{2}+\left|\sin \left(\theta_{j}-\theta_{3}+\epsilon\right)\right|^{\alpha} p_{3}, \quad j=1,2,3
\end{aligned}
$$


for small $\epsilon>0$. Adding the equations (3.1) and (3.2) respectively, we obtain

$$
\int_{[0, \pi)} \varphi(\xi) \tilde{\Gamma}(d \xi)=\sum_{j=1}^{3} \sum_{k=1}^{3}\left|\sin \left(\theta_{j}-\theta_{k}\right)\right|^{\alpha} p_{k}
$$

and

$$
\int_{[0, \pi)} \varphi_{\epsilon}(\xi) \tilde{\Gamma}(d \xi)=\sum_{j=1}^{3} \sum_{k=1}^{3} a_{j}\left|\sin \left(\theta_{j}-\theta_{k}+\epsilon\right)\right|^{\alpha} p_{k}
$$

for any $a_{1}, a_{2}, a_{3}$, where $\varphi(\xi)=\left|\sin \left(\theta_{1}-\xi\right)\right|^{\alpha}+\left|\sin \left(\theta_{2}-\xi\right)\right|^{\alpha}+\left|\sin \left(\theta_{3}-\xi\right)\right|^{\alpha}$ and $\varphi_{\epsilon}(\xi)=a_{1}\left|\sin \left(\theta_{1}+\epsilon-\xi\right)\right|^{\alpha}+a_{2}\left|\sin \left(\theta_{2}+\epsilon-\xi\right)\right|^{\alpha}+a_{3}\left|\sin \left(\theta_{3}+\epsilon-\xi\right)\right|^{\alpha}$. Now let us determine the coefficients $a_{1}, a_{2}, a_{3}$ so that $\varphi(\xi)=\varphi_{\epsilon}(\xi)$ at $\xi=\theta_{1}, \theta_{2}, \theta_{3}$. That is, determine $a_{1}, a_{2}, a_{3}$ satisfying the equation

$$
\begin{gathered}
\left(\begin{array}{ccc}
|\sin \epsilon|^{\alpha} & \left|\sin \left(\theta_{2}-\theta_{1}+\epsilon\right)\right|^{\alpha} & \left|\sin \left(\theta_{3}-\theta_{1}+\epsilon\right)\right|^{\alpha} \\
\left|\sin \left(\theta_{1}-\theta_{2}+\epsilon\right)\right|^{\alpha} & |\sin \epsilon|^{\alpha} & \left|\sin \left(\theta_{3}-\theta_{2}+\epsilon\right)\right|^{\alpha} \\
\left|\sin \left(\theta_{1}-\theta_{3}+\epsilon\right)\right|^{\alpha} & \left|\sin \left(\theta_{2}-\theta_{3}+\epsilon\right)\right|^{\alpha} & |\sin \epsilon|^{\alpha}
\end{array}\right)\left(\begin{array}{l}
a_{1} \\
a_{2} \\
a_{3}
\end{array}\right) \\
=\left(\begin{array}{l}
\left|\sin \left(\theta_{2}-\theta_{1}\right)\right|^{\alpha}+\left|\sin \left(\theta_{3}-\theta_{1}\right)\right|^{\alpha} \\
\left|\sin \left(\theta_{1}-\theta_{2}\right)\right|^{\alpha}+\left|\sin \left(\theta_{3}-\theta_{2}\right)\right|^{\alpha} \\
\left|\sin \left(\theta_{1}-\theta_{3}\right)\right|^{\alpha}+\left|\sin \left(\theta_{2}-\theta_{3}\right)\right|^{\alpha}
\end{array}\right) .
\end{gathered}
$$

Let $A_{\epsilon}(\epsilon \geq 0)$ be the determinant of the coefficient matrix in the left hand side. Then we see that $A_{\epsilon}$ is continuous in $\epsilon$ and $A_{0}=2 \mid \sin \left(\theta_{1}-\right.$ $\left.\theta_{2}\right)\left.\right|^{\alpha}\left|\sin \left(\theta_{2}-\theta_{3}\right)\right|^{\alpha}\left|\sin \left(\theta_{3}-\theta_{1}\right)\right|^{\alpha}>0$, so that we have $A_{\epsilon}>0$ for sufficiently small $\epsilon>0$. Thus $a_{1}, a_{2}, a_{3}$ exist uniquely for any fixed small $\epsilon>0$. For these $a_{1}, a_{2}, a_{3}$, we notice that the right hand sides of (3.3) and (3.4) are equal to each other and therefore

$$
\int_{[0, \pi)} \varphi(\xi) \tilde{\Gamma}(d \xi)=\int_{[0, \pi)} \varphi_{\epsilon}(\xi) \tilde{\Gamma}(d \xi)
$$

Next let us show that

$$
a_{j}=1-k_{j} \epsilon^{\alpha}+O\left(\epsilon^{2 \alpha \wedge 1}\right) \quad(j=1,2,3)
$$

for some $k_{j}>0$ as $\epsilon \rightarrow 0$. We show it only for $a_{1}$, as $a_{2}$ and $a_{3}$ are treated similarly. By Cramer's rule, we have

$$
a_{1}=\frac{1}{A_{\epsilon}}\left|\begin{array}{ccc}
\left|\sin \left(\theta_{2}-\theta_{1}\right)\right|^{\alpha}+\left|\sin \left(\theta_{3}-\theta_{1}\right)\right|^{\alpha} & \left|\sin \left(\theta_{2}-\theta_{1}+\epsilon\right)\right|^{\alpha} & \left|\sin \left(\theta_{3}-\theta_{1}+\epsilon\right)\right|^{\alpha} \\
\left|\sin \left(\theta_{1}-\theta_{2}\right)\right|^{\alpha}+\left|\sin \left(\theta_{3}-\theta_{2}\right)\right|^{\alpha} & |\sin \epsilon|^{\alpha} & \left|\sin \left(\theta_{3}-\theta_{2}+\epsilon\right)\right|^{\alpha} \\
\left|\sin \left(\theta_{1}-\theta_{3}\right)\right|^{\alpha}+\left|\sin \left(\theta_{2}-\theta_{3}\right)\right|^{\alpha} & \left|\sin \left(\theta_{2}-\theta_{3}+\epsilon\right)\right|^{\alpha} & |\sin \epsilon|^{\alpha}
\end{array}\right| .
$$


Therefore we obtain

$$
\begin{aligned}
a_{1} & =\frac{A_{0}+c_{1} \epsilon^{\alpha}+O\left(\epsilon^{2 \alpha \wedge 1}\right)}{A_{0}+b \epsilon^{\alpha}+O\left(\epsilon^{2 \alpha \wedge 1}\right)}=\frac{1+\frac{c_{1}}{A_{0}} \epsilon^{\alpha}+O\left(\epsilon^{2 \alpha \wedge 1}\right)}{1+\frac{b}{A_{0}} \epsilon^{\alpha}+O\left(\epsilon^{2 \alpha \wedge 1}\right)} \\
& =\left(1+\frac{c_{1}}{A_{0}} \epsilon^{\alpha}+O\left(\epsilon^{2 \alpha \wedge 1}\right)\right)\left(1-\frac{b}{A_{0}} \epsilon^{\alpha}-O\left(\epsilon^{2 \alpha \wedge 1}\right)\right) \\
& =1-\frac{b-c_{1}}{A_{0}} \epsilon^{\alpha}+O\left(\epsilon^{2 \alpha \wedge 1}\right),
\end{aligned}
$$

where

$$
\begin{aligned}
& b=-\left\{\left(\left|\sin \left(\theta_{2}-\theta_{1}\right)\right|^{\alpha}\right)^{2}+\left(\left|\sin \left(\theta_{3}-\theta_{1}\right)\right|^{\alpha}\right)^{2}+\left(\left|\sin \left(\theta_{3}-\theta_{2}\right)\right|^{\alpha}\right)^{2}\right\}, \\
& c_{1}=-\left|\sin \left(\theta_{2}-\theta_{1}\right)\right|^{\alpha}\left(\left|\sin \left(\theta_{1}-\theta_{2}\right)\right|^{\alpha}+\left|\sin \left(\theta_{3}-\theta_{2}\right)\right|^{\alpha}\right) \\
&-\left|\sin \left(\theta_{3}-\theta_{1}\right)\right|^{\alpha}\left(\left|\sin \left(\theta_{1}-\theta_{3}\right)\right|^{\alpha}+\left|\sin \left(\theta_{2}-\theta_{3}\right)\right|^{\alpha}\right) .
\end{aligned}
$$

We see that

$b-c_{1}=\left|\sin \left(\theta_{3}-\theta_{2}\right)\right|^{\alpha}\left(\left|\sin \left(\theta_{2}-\theta_{1}\right)\right|^{\alpha}+\left|\sin \left(\theta_{3}-\theta_{1}\right)\right|^{\alpha}-\left|\sin \left(\theta_{3}-\theta_{2}\right)\right|^{\alpha}\right)>0$

and hence we obtain the formula $(3.6)$ by setting $k_{1}=\left(b-c_{1}\right) / A_{0}$.

Now let us show that $\varphi_{\epsilon}(\xi)<\varphi(\xi)$ at $\xi \neq \theta_{1}, \theta_{2}, \theta_{3}$ for sufficiently small $\epsilon$. We show it on $\left(\theta_{1}, \theta_{2}\right)$. (The proof is similar on $\left(\theta_{2}, \theta_{3}\right)$ or $\left(\theta_{3}, \pi\right) \cup\left[0, \theta_{1}\right)$.) We note that $\frac{d}{d \xi}\left|\sin \left(\theta_{j}-\xi\right)\right|^{\alpha} \rightarrow-\infty$ as $\xi \rightarrow \theta_{j}-0, \frac{d}{d \xi}\left|\sin \left(\theta_{j}-\xi\right)\right|^{\alpha} \rightarrow \infty$ as $\xi \rightarrow \theta_{j}+0$ and $\frac{d^{2}}{d \xi^{2}}\left|\sin \left(\theta_{j}-\xi\right)\right|^{\alpha}<0$ at $\xi \neq \theta_{j}$ for $j=1,2,3$. Therefore we have $\varphi_{\epsilon}(\xi)<\varphi(\xi)$ on $\left(\theta_{1}, \theta_{1}+\epsilon\right]$ for sufficiently small $\epsilon$ by $\varphi_{\epsilon}\left(\theta_{1}\right)=\varphi\left(\theta_{1}\right)$. And we have

$$
\begin{aligned}
& a_{1}\left|\sin \left(\theta_{1}+\epsilon-\xi\right)\right|^{\alpha}<\left|\sin \left(\theta_{1}+\epsilon-\xi\right)\right|^{\alpha}<\left|\sin \left(\theta_{1}-\xi\right)\right|^{\alpha} \\
& a_{3}\left|\sin \left(\theta_{3}+\epsilon-\xi\right)\right|^{\alpha}=\left(1-k_{3} \epsilon^{\alpha}+O\left(\epsilon^{2 \alpha \wedge 1}\right)\right)\left(\left|\sin \left(\theta_{3}-\xi\right)\right|^{\alpha}+O(\epsilon)\right) \\
&<\left|\sin \left(\theta_{3}-\xi\right)\right|^{\alpha}
\end{aligned}
$$

on $\left(\theta_{1}+\epsilon, \theta_{2}\right)$ for sufficiently small $\epsilon$. Let $\delta$ be $\delta=2 \alpha \epsilon^{1-\alpha} / k_{2}$. (We note that $\delta \rightarrow 0$ as $\epsilon \rightarrow 0$.) Then we obtain

$$
\left|\sin \left(\theta_{2}+\epsilon-\xi\right)\right|^{\alpha}<\left|\sin \left(\theta_{2}-\xi\right)\right|^{\alpha}+\epsilon \alpha\left|\sin \left(\theta_{2}-\xi\right)\right|^{\alpha-1} \cos \left(\theta_{2}-\xi\right)
$$


for $\theta_{1}+\epsilon<\xi \leq \theta_{2}-\delta$ by mean value theorem and we have

$$
\begin{aligned}
a_{2} \mid & \left.\sin \left(\theta_{2}+\epsilon-\xi\right)\right|^{\alpha} \\
& <\left(1-k_{2} \epsilon^{\alpha}+O\left(\epsilon^{2 \alpha \wedge 1}\right)\right)\left(\left|\sin \left(\theta_{2}-\xi\right)\right|^{\alpha}+\epsilon \alpha\left|\sin \left(\theta_{2}-\xi\right)\right|^{\alpha-1}\right) \\
& =\left|\sin \left(\theta_{2}-\xi\right)\right|^{\alpha}+\epsilon \alpha\left|\sin \left(\theta_{2}-\xi\right)\right|^{\alpha-1}-k_{2} \epsilon^{\alpha}\left|\sin \left(\theta_{2}-\xi\right)\right|^{\alpha}+O\left(\epsilon^{2 \alpha \wedge 1}\right) \\
& \leq\left|\sin \left(\theta_{2}-\xi\right)\right|^{\alpha}+\epsilon \alpha \delta^{\alpha-1}-k_{2} \epsilon^{\alpha} \delta^{\alpha}+O\left(\epsilon^{2 \alpha \wedge 1}\right) \\
& =\left|\sin \left(\theta_{2}-\xi\right)\right|^{\alpha}-2^{\alpha-1} \alpha^{\alpha} k_{2}^{1-\alpha} \epsilon^{-\alpha^{2}+2 \alpha}+O\left(\epsilon^{2 \alpha \wedge 1}\right) \\
& <\left|\sin \left(\theta_{2}-\xi\right)\right|^{\alpha}
\end{aligned}
$$

on $\left(\theta_{1}+\epsilon, \theta_{2}-\delta\right]$ for sufficiently small $\epsilon$. Therefore we find $\varphi_{\epsilon}(\xi)<\varphi(\xi)$ on $\left(\theta_{1}+\epsilon, \theta_{2}-\delta\right]$. And we see $\varphi_{\epsilon}(\xi)<\varphi(\xi)$ on $\left(\theta_{2}-\delta, \theta_{2}\right)$ for sufficiently small $\epsilon$ by $\varphi_{\epsilon}\left(\theta_{2}\right)=\varphi\left(\theta_{2}\right)$. Therefore we have $\varphi_{\epsilon}(\xi)<\varphi(\xi)$ on $\left(\theta_{1}, \theta_{2}\right)$.

Hence, by $(3.5)$, we conclude that $\left.\tilde{\Gamma}\right|_{[0, \pi)}$ is concentrated on $\left\{\theta_{1}, \theta_{2}, \theta_{3}\right\}$. We can easily verify that $\tilde{\Gamma}\left(\left\{\theta_{j}\right\}\right)=p_{j}(j=1,2,3)$.

Remark 3.2. This proposition is also true if we replace $[\pi / 2, \pi)$ in the statement with $[\pi / 2, \pi) \cup\{0\}$.

Remark 3.3. We note that Proposition 3.1 fails when $\alpha=1$ and $\left.\Gamma\right|_{[0, \pi)}$ is concentrated on $\{0, \pi / 2\}$. For example, let us define $X=\left(X_{1}, X_{2}\right)$ and $\tilde{X}=\left(\tilde{X}_{1}, \tilde{X}_{2}\right)$ such that

$$
\begin{aligned}
& E \exp \left(i\left(z_{1} X_{1}+z_{2} X_{2}\right)\right)=\exp \left(-\left(\left|z_{1}\right|+\left|z_{2}\right|\right)\right) \\
& E \exp \left(i\left(z_{1} \tilde{X}_{1}+z_{2} \tilde{X}_{2}\right)\right)=\exp \left(-\left(\frac{1}{3}\left|z_{1}+2 z_{2}\right|+\frac{1}{3}\left|2 z_{1}+z_{2}\right|\right)\right) .
\end{aligned}
$$

Then $z_{1} \tilde{X}_{1}+z_{2} \tilde{X}_{2} \stackrel{d}{=} z_{1} X_{1}+z_{2} X_{2}$ for $z_{1} z_{2} \geq 0$ although $\tilde{X} \stackrel{d}{\neq} X$.

Conjecture 3.4. It seems that Proposition 3.1 remains true if we replace "at most three points" by "a finite number of points".

Similarly to the extension of Theorem 2.1 to Proposition 2.2, we have the following proposition.

Proposition 3.5. Let $X=\left(X_{1}, X_{2}\right)$ be a 2-dimensional symmetric $\alpha$-stable random variable $(0<\alpha<1)$ whose spectral measure $\Gamma$ satisfies that $\left.\Gamma\right|_{[0, \pi)}$ is concentrated on at most three points on $\left[\theta_{1}, \theta_{2}\right]\left(0 \leq \theta_{1}<\right.$ $\left.\theta_{2}<\pi\right)$. Suppose that a 2-dimensional symmetric $\alpha$-stable random variable $\tilde{X}=\left(\tilde{X}_{1}, \tilde{X}_{2}\right)$ satisfies the condition that

$$
z_{1} \tilde{X}_{1}+z_{2} \tilde{X}_{2} \stackrel{d}{=} z_{1} X_{1}+z_{2} X_{2} \quad \text { if } \arg \left(z_{1}, z_{2}\right) \in\left[\theta_{1}+\pi / 2, \theta_{2}+\pi / 2\right]
$$


Then $\tilde{X} \stackrel{d}{=} X$.

Acknowledgement. The author would like to thank the referee for useful comments which improved this paper.

\section{REFERENCES}

[1] D. J. Marcus, Non-stable laws with all projections stable, Z. Wahrsch. verw. Gebiete, 64 (1983), 139-156.

[2] G. Samorodnitsky and M. S. Taqqu, Stable non-Gaussian random processes, Chapman \& Hall, New York, 1994.

Niihama National College of Technology

Niihama, 792-8580

Japan

kojo@sci.niihama-nct.ac.jp 